\title{
Analysis of the Spatial Distribution Characteristics of Abnormal Bone Density in Male Long-Distance Runners from Different Regions in China
}

\author{
Longxing Fan1", Wenjie Zhang2 ${ }^{2}$, Huanhuan Cui ${ }^{3}$, Yanqing Liu ${ }^{3}$, Ziquan Liu ${ }^{3 \#}$ \\ ${ }^{1}$ Ministry of Education Key Laboratory for Analytical Science of Food Safety and Biology, Fujian Provincial Key Laboratory of \\ Analysis and Detection Technology for Food Safety, College of Chemistry, Fuzhou University, Fuzhou, China \\ ${ }^{2}$ Tianjin Center for Disease Control and Prevention, Tianjin, China \\ ${ }^{3}$ Institute of Disaster Medicine, Tianjin University, Tianjin, China \\ Email:494071841@qq.com,615340858@qq.com, "liuziquanwj@163.com
}

How to cite this paper: Fan, L.X., Zhang, W.J., Cui, H.H., Liu, Y.Q. and Liu, Z.Q. (2021) Analysis of the Spatial Distribution Characteristics of Abnormal Bone Density in Male Long-Distance Runners from Different Regions in China. Open Journal of Epidemiology, 11, 80-91.

https://doi.org/10.4236/ojepi.2021.111008

Received: October 16, 2020

Accepted: February 2, 2021

Published: February 5, 2021

Copyright $\odot 2021$ by author(s) and Scientific Research Publishing Inc. This work is licensed under the Creative Commons Attribution International License (CC BY 4.0).

http://creativecommons.org/licenses/by/4.0/

(c) (i) Open Access

\begin{abstract}
Objective: To investigate the occurrence pattern of abnormal bone density in male long-distance runners from several different regions of China, and provide a basis for elucidating the influences of geo-environmental differences on bone density. Methods: We employed a set of well-designed exclusioninclusion criteria to recruit study subjects, in which compounding factors were managed and regional environmental traits were fully incorporated. WHO (World Health Organization) criteria for the diagnosis of osteoporosis were then used to examine the subjects to determine occurrence of abnormal bone density. The resulting data were analyzed using methods of spatial statistics, which included several approaches, such as spatial autocorrelation, hot spot analysis, and Geodetector Software analysis, to depict and analyze the spatial distribution of abnormal bone density in male athletes from different regions in China, thereby investigating the influences of geo-environmental factors (e.g., temperature, humidity, and altitude) on bone density. Results: A total of 685 subjects were effectively examined in this study, including 486 with normal bone density, 185 with osteopenia, and 14 with osteoporosis. Spatial distribution analysis revealed that the distribution of subjects with abnormal bone density overall exhibited a pattern indicating that the level of abnormal bone density in the eastern regions was higher than that in the western regions and that the levels of abnormal bone density in the southern and northern regions were higher than that in the middle regions. Spatial autocorrelation analysis revealed a Moran's $I=0.136, Z$-score $=1.114$, and $P$
\end{abstract}

${ }^{\star}$ Longxing Fan, Wenjie Zhang contributed equally to this work; ${ }^{\sharp}$ Corresponding author. 
value $=0.265$ and indicated that the athletes with abnormal bone density were randomly distributed in each region. Hot spot analysis revealed that Tibet and Qinghai displayed distributions of cold spots. Geodetector Software analysis yielded a $Q$ value for annual average temperature of 1.000 and a corresponding $P$ value of 0.000 , and the results revealed that temperature significantly affected bone density and that altitude, relative humidity, sunlight hours, and temperature variations displayed synergistic effects on bone density and could diminish the influences of temperature on bone density. Conclusion: Our data revealed that different regions displayed different distribution patterns of abnormal bone density such that the level in the eastern regions was higher than that in the western ones and that the levels in the southern and northern regions were higher than that in the middle regions; specifically, the provinces of Yunnan, Heilongjiang, Hainan, and Inner Mongolia had high rates of abnormal bone density, whereas Tibet and Qinghai had relatively good conditions of bone density. Our data suggested that suitable temperature changes and appropriate levels of temperature variations can decrease the occurrence rates of osteopenia and osteoporosis.

\section{Keywords}

Spatial Epidemiology, Regions, Athletes, Bone Density

\section{Introduction}

Osteoporosis is a systemic bone disease characterized by bone loss, osteopenia, and bone microstructural changes that result from multiple factors [1] [2] [3]. Osteoporosis causes body pain, spinal deformity, and severe fractures and, as such, greatly affects people's health and quality of life [4]. It is well known that osteoporosis has a relatively high prevalence in menopausal women and elderly people. However, in recent years, coinciding with increased public attention on the bone disease, it has come to our attention that high-risk populations also include adolescents in exuberant development, athletes with intensive training, and workers under high physical load [5] [6] [7] [8].

Currently, well-recognized risk factors of osteoporosis mainly include age, race, gender, alteration of sexual function, abnormal body weight, nutritional abnormalities, and medication [9] [10]. However, few studies have been dedicated to examining the correlations between osteoporosis and environmental factors, such as temperature, humidity, and altitude [11] [12]. China has a vast territory that harbors an extraordinary diversity of geo-environmental traits, which is conducive for investigating the relationships between the natural environment and diseases [13]. In addition, China has a large population that accommodates a large pool of occupational individuals, which is convenient for recruiting study subjects. Here, we investigated the influence of environmental factors on the bone density of populations by analyzing athletes from different regions that have distinct environmental traits and employing newly developed geological 
surveillance technologies, including Geographic Information Systems (GIS), Global Positioning Systems (GPS), and Remote Sensing (RS) [14]. The results enabled us to examine the influences of environmental factors on the bone density of populations.

\section{Data and Methods}

\section{Data sources}

Selection of Regions and Sources of Geological Information: We chose provincial capitals that feature high temperatures, high humidity, high altitude, and low temperature, specifically, the provincial seats of Sichuan, Tibet, Yunnan, Hainan, Heilongjiang, Inner Mongolia, and Xinjiang. The provinces were classified based on the four aforementioned environmental traits. We then collected geological and climate data, including altitude, sunlight hours, 1981-2010 annual average temperature, and 1981-2010 annual average relative humidity. All data were from the cloud data center of the Chinese Academy of Meteorological Sciences (http://data.cma.cn/).

Recruitment Criteria of the Subjects. This study was conducted in accordance with the Declaration of Helsinki. All study participants were fully aware of the study content and signed the informed consent. The inclusion criteria were as follows: 1) male, 2) aged 18 - 26 years old, 3) being in long-term (>2 years) training with local sports schools or provincial sports teams, 4) long-distance running over $5 \mathrm{~km}$ daily, 5) scientifically designed meals, 6) history of health, with no severe bone disease in the last two years, and 7) no history of other chronic disease. The exclusion criteria were as follows: 1) history of chronic disease and corresponding medication, 2) obesity, hypertension, or diabetes, 3) retired athletes, and 4) disabled athletes.

\section{Study methods}

Examination Methods: Digital radiography-based uptake was employed to determine the bone densities of the second, third, and fourth fingers of the dominant hand, which were measured using a Metriscan Bone Densitometer (Alara, U.S.A.). Diagnosis was conducted following the corresponding WHO criteria: normal $(T$ value $>-1)$, osteopenia $(-1>T$ value $>-2.5)$, and osteoporosis $(T$ value $<-2.5)$.

Statistical Analyses. Exploratory Spatial Data Analysis (ESDA) and spatial stratified heterogeneity were the major methods used in this study. Both methods can quantitatively reveal the spatial relationship and aggregation of high-risk factors by calculating the relevant traits. Correspondingly, they can elucidate spatial stratified heterogeneity and explore dictating factors of disease, thereby providing evidence to understand the trends of disease-influencing factors in monitored regions. In this study, the methods, namely, Moran's I and Getis-Ord $\mathrm{Gi}^{*}$, were employed to analyze the traits of the participants and to elucidate their spatial aggregation under a significance level of $\alpha=0.05$. The resulting data were visualized using the following equations [15] [16]. 


$$
\begin{gathered}
I=\frac{n \sum_{i=1}^{n} \sum_{j=1}^{n}\left(x_{i}-\bar{x}\right)\left(x_{j}-\bar{x}\right)}{\left(\sum_{i=1}^{n} \sum_{j=1}^{n} W_{i j}\right) \sum_{i=1}^{n}\left(x_{i}-\bar{x}\right)^{2}} \\
\bar{x}=\frac{1}{n} \sum_{i=1}^{n} x_{i}
\end{gathered}
$$

in which $n$ represents the count of observed traits, $x_{i}$ represents the observed value at location $i, x_{j}$ represents the observed value at location $j, j \neq i, \bar{x}$ represents the mean of all observed values at all $n$ locations, and $W_{i j}$ represents the spatial weight matrix that measures the mutual influences between locations $i$ and $j$ and has multiple forms.

$$
\begin{gathered}
G_{i}^{*}=\frac{\sum_{j=1}^{n} W_{i j}(d) x_{j}-W_{j}^{*} \bar{x}}{S \sqrt{\left(n S_{1 j}-W_{i}^{* 2}\right) /(n-1)}} \\
W_{i}^{*}=\sum_{j=1}^{n} W_{i j}, \quad S=\sqrt{\frac{1}{n} \sum_{i=1}^{n}\left(x_{i}-\bar{x}\right)^{2}}, \quad S_{1 j}=\sum_{j=1}^{n} W_{i j}^{2}
\end{gathered}
$$

in which $G_{i}^{*}$ represents the level of correlation between observed values at location $i$ and observed values of all locations $j$ that are within a distance $d$ from location $i, W_{i j}(d)$ represents the neighboring spatial weight matrix within distance $d$, and $S$ represents the standard deviation of each observed value. In addition, expected values and variance could also be calculated based on $G_{i}^{*}$, whereby $Z$ was derived for the comparison of threshold values and subsequent statistical analyses.

Analysis Software: Office Excel 2007, ArcGIS 10.0, and GeoDetector were used for data analyses in this study.

\section{Results}

\section{General data information}

All participants were athletes from different provincial capitals. Background meteorological data were from the weather stations of the sampling cities. Non-station observatory data were used to generate fitting results, which were straightforward. The geo-environmental data of different provinces, which were from weather stations, are summarized in Table 1 . The background meteorological data of the sampling cities are shown in Figure 1. A total of 685 participants were effectively examined in this study, including 486 subjects with normal bone density, 185 subjects with osteopenia, and 14 subjects with osteoporosis. The bone density test results for athletes from different provinces are compiled in Table 2, and the compositions of the three bone density categories are shown in Figure 2.

\section{Spatial analysis results}

In this study, we combined the participants with osteopenia and osteoporosis into a single group of abnormal bone density to calculate the occurrence, which 
Table 1. Geo-environmental data derived from local weather stations in different provinces.

\begin{tabular}{cccccc}
\hline $\begin{array}{c}\text { Province } \\
\text { (sampling site, weather stations) }\end{array}$ & $\begin{array}{c}\text { Annual average } \\
\text { temperature } \\
\left({ }^{\circ} \mathrm{C}\right)\end{array}$ & $\begin{array}{c}\text { Altitude } \\
(\mathrm{m})\end{array}$ & $\begin{array}{c}\text { Average } \\
\text { humidity } \\
(\%)\end{array}$ & $\begin{array}{c}\text { Average } \\
\text { temperature } \\
\text { variation }\left({ }^{\circ} \mathrm{C}\right)\end{array}$ & $\begin{array}{c}\text { Sunlight } \\
\text { hours }\end{array}$ \\
\hline Sichuan (Chengdu, Wenjiang)
\end{tabular}

Note. All data were from the cloud data center of the Chinese Academy of Meteorological Sciences (http://data.cma.cn/); Significance level of the GeoDetector analysis: $\alpha=0.05$.
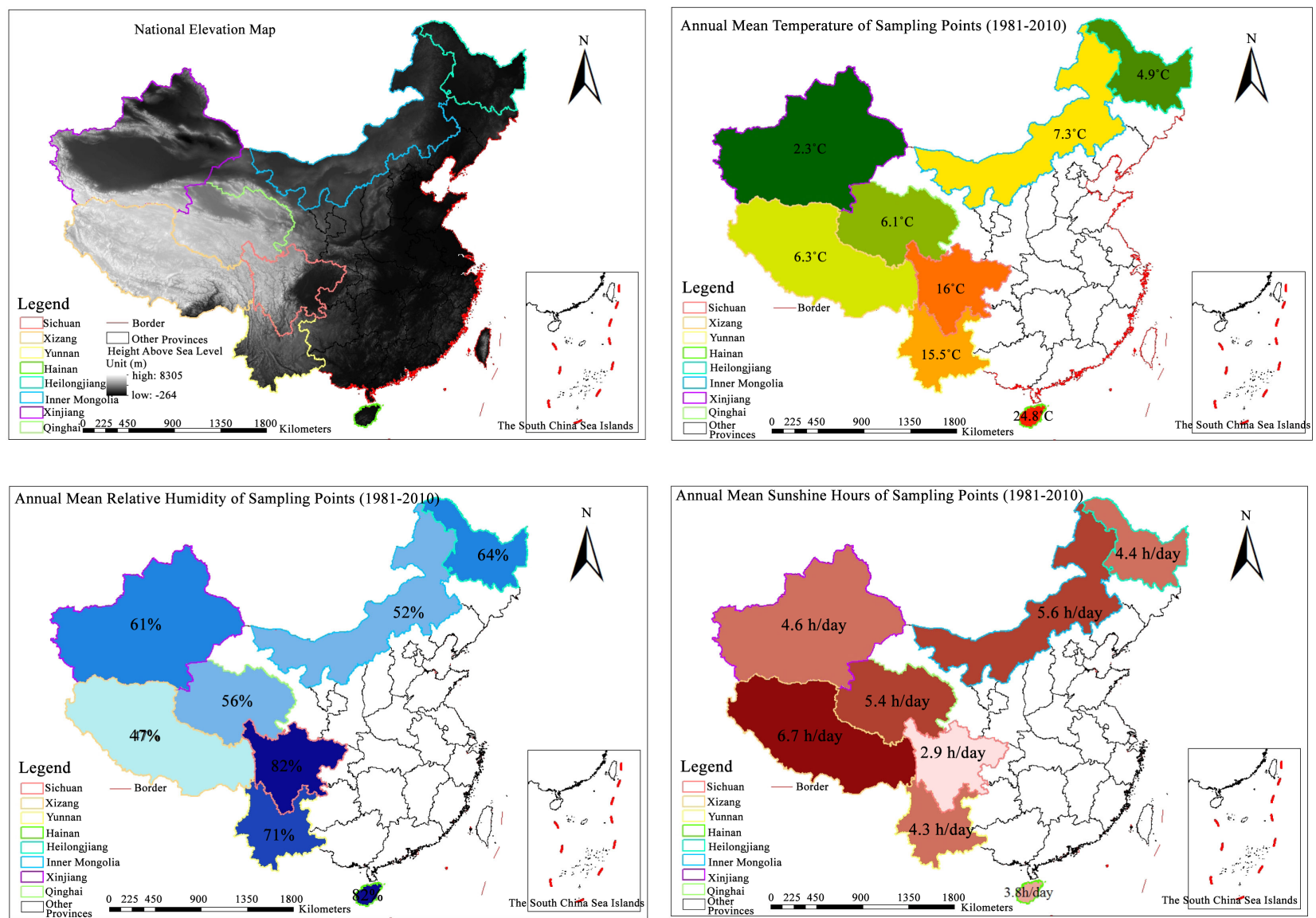

Figure 1. Background meteorological data for the sampling cities. 


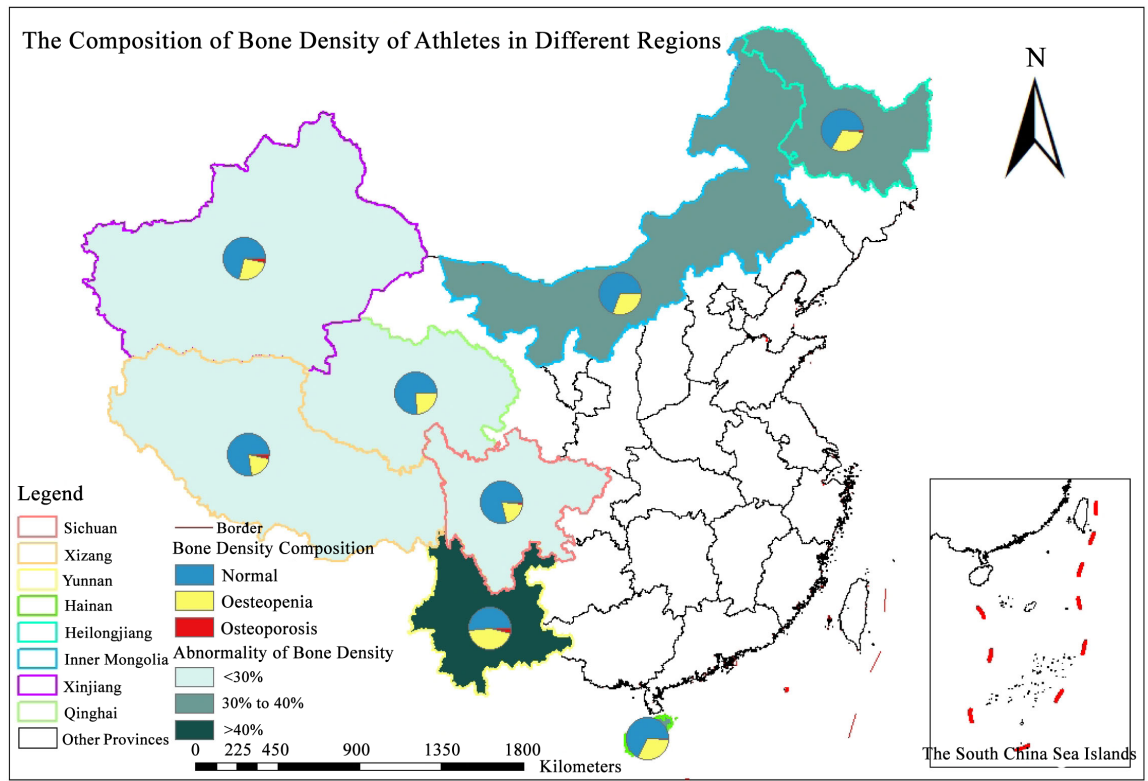

Figure 2. Pie chart of different bone density categories of athletes from different provinces.

Table 2. Bone density test results for athletes from different provinces.

\begin{tabular}{ccccccccc}
\hline & & \multicolumn{2}{c}{ Normal } & \multicolumn{2}{c}{ Osteopenia } & \multicolumn{2}{c}{ Osteoporosis } & \multirow{2}{*}{ Province } \\
\cline { 3 - 6 } & Subjects $(\mathrm{n})$ & $\mathrm{n}$ & $\%$ & $\mathrm{n}$ & $\%$ & $\mathrm{n}$ & $\%$ & \\
\hline Sichuan & 113 & 89 & 0.79 & 22 & 0.19 & 2 & 0.02 & $-0.35 \pm 0.94$ \\
Tibet & 131 & 102 & 0.78 & 25 & 0.19 & 4 & 0.03 & $-0.08 \pm 1.26$ \\
Yunnan & 67 & 35 & 0.52 & 29 & 0.43 & 3 & 0.04 & $-0.92 \pm 1.03$ \\
Hainan & 80 & 54 & 0.68 & 25 & 0.31 & 1 & 0.01 & $-0.48 \pm 0.98$ \\
Heilongjiang & 98 & 65 & 0.66 & 31 & 0.32 & 2 & 0.02 & $-0.46 \pm 1.07$ \\
Inner Mongolia & 55 & 38 & 0.69 & 17 & 0.31 & 0 & 0.00 & $-0.36 \pm 0.99$ \\
Xinjiang & 71 & 50 & 0.70 & 19 & 0.27 & 2 & 0.03 & $-0.46 \pm 1.11$ \\
Qinghai & 70 & 53 & 0.76 & 17 & 0.24 & 0 & 0.00 & $-0.52 \pm 0.87$ \\
Sum & 685 & 486 & 0.71 & 185 & 0.27 & 14 & 0.02 & $-0.42 \pm 1.07$ \\
\hline
\end{tabular}

was then used for subsequent spatial analyses, with the detailed results shown in Figure 3. Spatial trend analysis (Figure 3(a)) revealed that overall, the prevalence of abnormal bone density of athletes in eastern regions was higher than that in western regions, and the prevalence in the southern and northern regions was higher than that in the middle regions. Autocorrelation (Moran's I) analysis revealed Moran's $I=0.136, Z$-score $=1.114$, and $P$ value $=0.265$ (Figure $3(\mathrm{~b})$ ), indicating that there were no significant differences between the data distribution and the random model. In other words, the cases of abnormal bone density in athletes were randomly distributed in each province, and there was no correlation between the occurrence and any particular region. Cluster and outlier analysis (Anselin Local Moran's I) generated similar results (Figure 3(c)), 

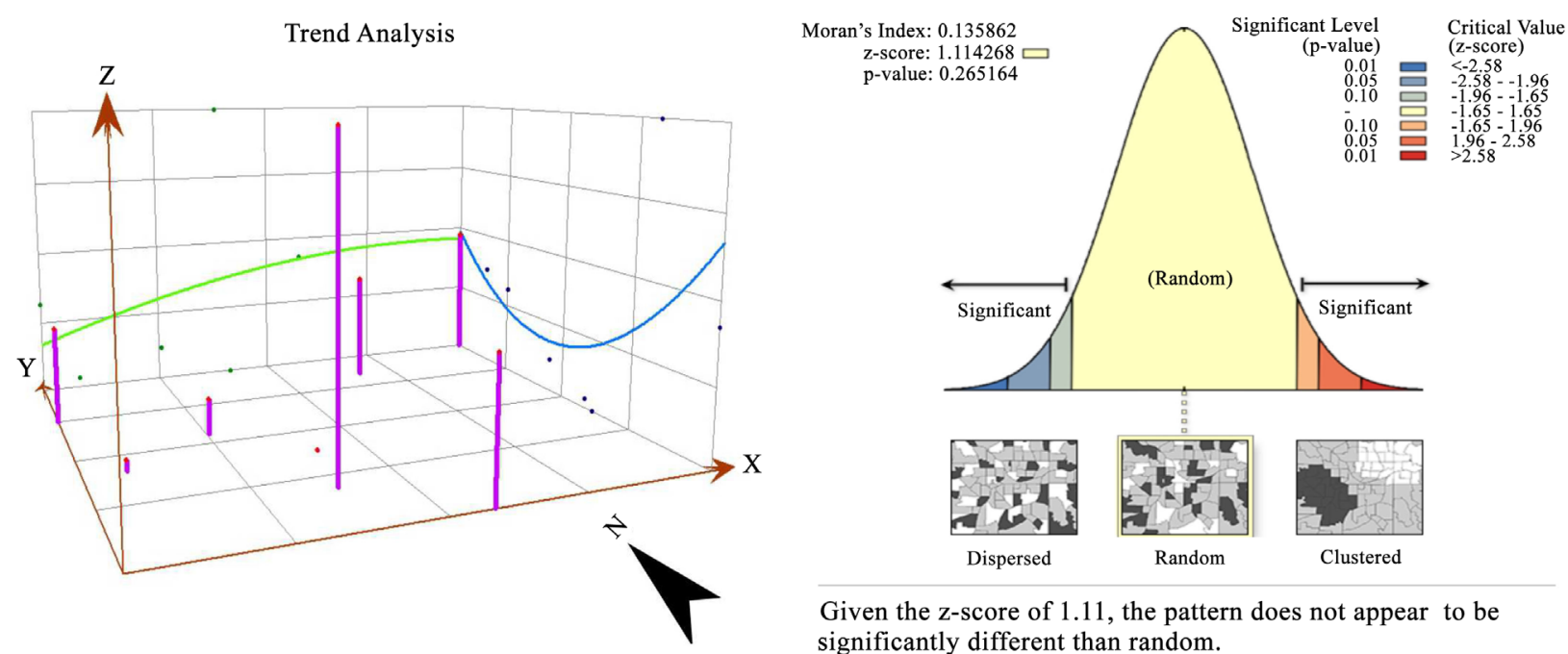

Given the z-score of 1.11 , the pattern does not appear to be significantly different than random.
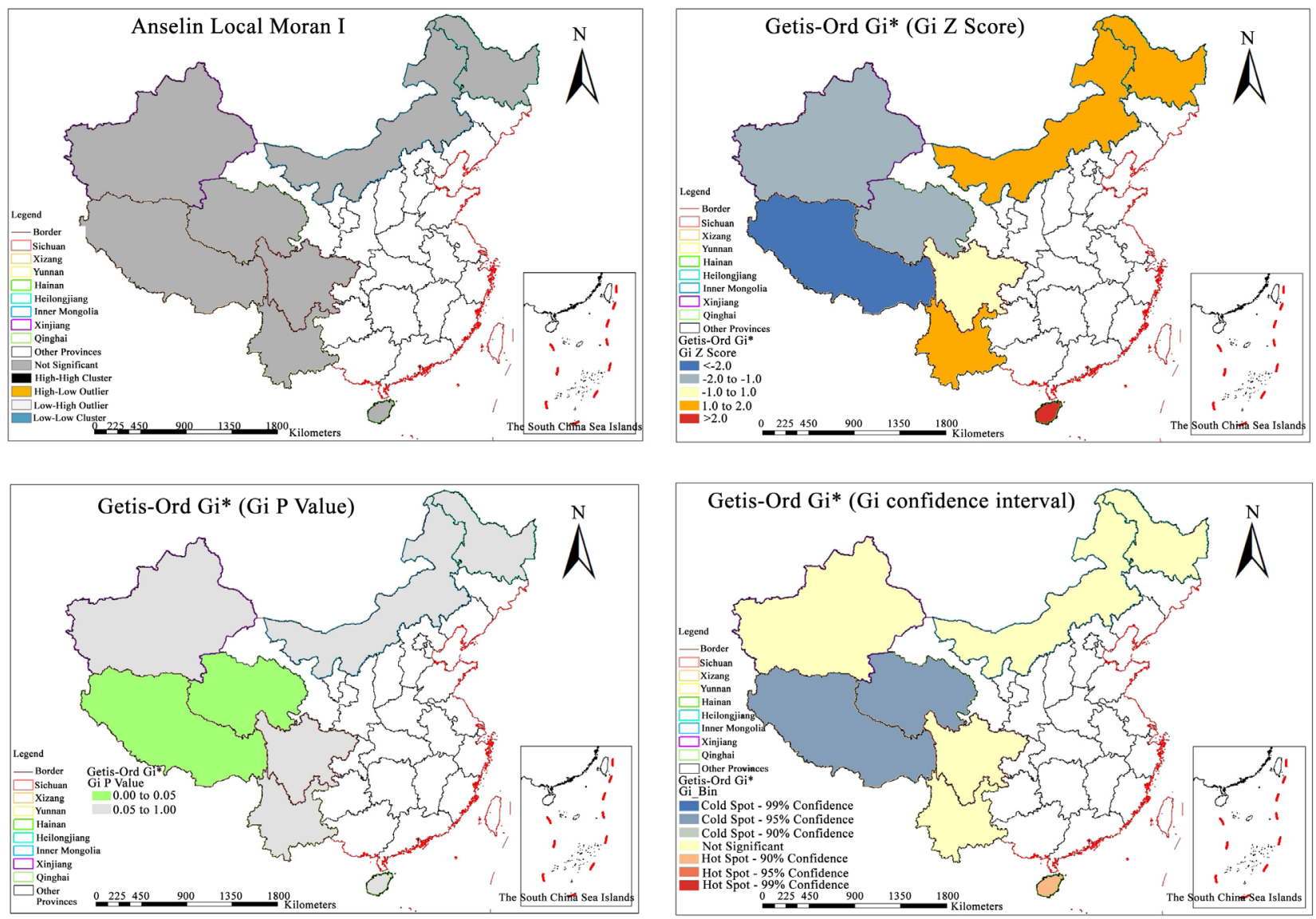

Figure 3. Spatial statistical analysis results.

further corroborating the conclusion that the occurrence of abnormal bone density in athletes displayed no spatial clustering and was randomly distributed. Hot spot analysis (Getis-Ord Gi*) revealed that Tibet and Qinghai belonged to cold spots (Figure 3(d), Figure 3(e), Figure 3(f)), or that the occurrences of abnormal bone density in the two regions were below the level of mathematical prediction. 


\section{GeoDetector analysis results}

GeoDetector was then used to perform analyses of spatial stratified heterogeneity, whereby the influences of temperature, altitude, relative humidity, temperature variation, and sunlight hours on abnormal bone density were investigated. The results of factor detector analysis indicated that temperature displayed the most prominent effects on bone density (Table 1). The results of interactive detector analysis indicated that altitude, relative humidity, sunlight hours, and temperature variation could all diminish the influences of temperature on bone density and that there were synergistic effects among altitude, relative humidity, sunlight hours, and temperature variation (Figure 4).

\section{Discussion}

As living standards improve, increasingly more people have begun to pay close attention to the influences of exercise on the body. Athletes are professional sportsmen who have better physical fitness than general populations; they have relatively stable physical indicators and are therefore good study subjects for examining the relationships between the body and sports. Bone strength directly affects an athlete's career, and maintaining solid bones is the physical foundation of most sportsmen. Bone density is the most simple and objective indicator to measure bone strength. In recent years, rapid advancements in nutriology and sports medicine have enabled scientists and physicians to identify many factors affecting bone density, which can be organized into four categories: body factors, nutrition factors, disease factors, and environmental factors. Currently, the body factors that have been validated include gender, age, and hormone levels. Peak bone density occurs when a person is between 20 and 30 years old and is slightly different between men and women. This period also represents the golden phase of most athletes [17]. With regard to nutrition factors, the most important ones are calcium intake level and vitamin D intake level and biosynthesis activity, although the absorption of other nutrients also affects the uptake and metabolism

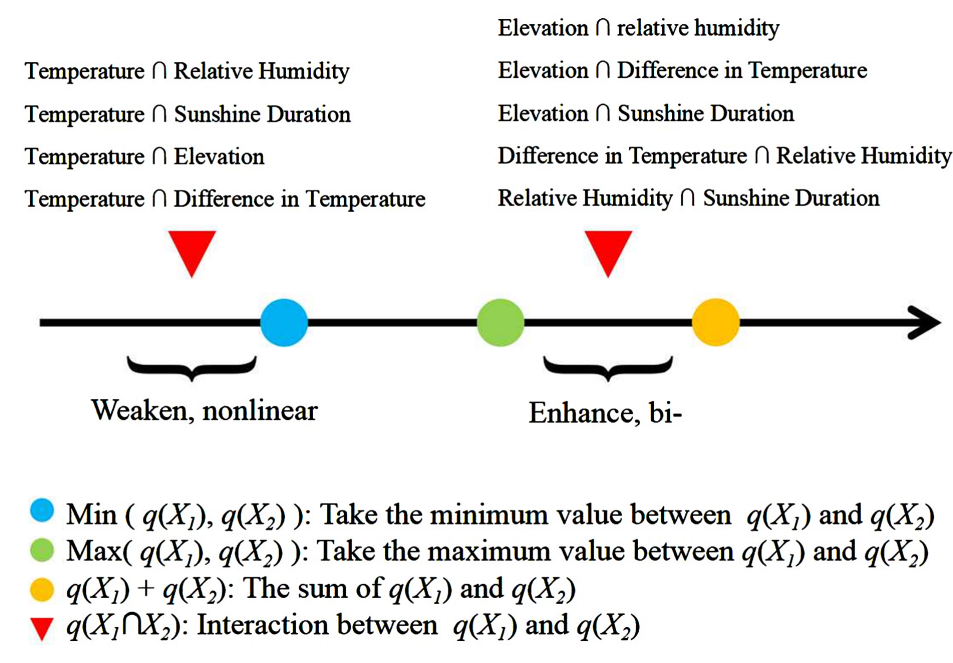

Figure 4. Analytic results of interactive detectors. 
of calcium [18]. Among disease factors, diabetes is a well-recognized illness that can alter bone density [19]. It has been reported that sunlight exposure is a major environmental factor affecting bone density, but thus far, the contributions of other environmental factors have been overlooked [20].

In this study, we recruited male long-distance runners to examine the relationship between various geo-environmental factors and abnormal bone density. We introduced a set of exclusion-inclusion criteria to minimize the interference of compounding factors, such as gender, age, nutrition, and disease, and to ensure that the study subjects were well-matched for these factors. Correspondingly, the participants were only differentiated by the geo-environmental factors; therefore, our results were convincing. In this study, we discovered apparent differences in occurrences of abnormal bone density between different regions such that the occurrences in the eastern regions were higher than those in the western ones, and the occurrences in the southern and northern regions were higher than those in the middle ones; specifically, the provinces of Yunnan, Heilongiiang, Hainan, and Inner Mongolia had high rates of abnormal bone density, which was reported by our group in a previous study [21]. In addition, spatial autocorrelation analysis revealed that occurrences of abnormal bone density in different regions showed no apparent spatial correlation, instead exhibiting a random distribution. In other words, cases of abnormal bone density in different regions were independent events, and there were interactions or influences among the regions. Hot spot analysis revealed that there were cold spots in Tibet and Qinghai, meaning that the actual occurrences of abnormal bone density were below the theoretically predicted values; thus, the conditions were better than those in the other regions. geodetector analysis was then conducted to analyze the relationship between environmental factors and abnormal bone density and revealed that temperature variation had the most prominent influence on this condition. Taking into account our clinical experience and previous reports (citations), we argued that suitable temperature variations can stimulate calcium intake, thereby augmenting bone structure and reducing the occurrence of abnormal bone density.

This study features the following advantages. First, we recruited athletes in different regions with a set of well-designed exclusion-inclusion criteria using appropriate tests, if applicable, thereby removing compounding factors such as gender, age, nutrition, and disease. Correspondingly, the subjects were only differentiated by the examined geo-environmental factors, thus ensuring the reliability of our results. Second, we employed spatial statistical analyses and spatial epidemiology approaches to investigate the influence of geo-environmental elements on a disease. In comparison with traditional statistical methods, this new strategy takes full advantage of spatial factors, which is conducive for big data-based applications.

During the course of this study, we were confronted with some challenges. Based on the results of statistical analyses, we considered that gender, nutrition, and disease were the most significant factors affecting bone density; in particu- 
lar, differences in nutrition uptake may directly contribute to alterations in bone density. Although competitive research based on spatial statistics revealed a connection between temperature and bone density, no apparent association was found for other geo-environmental factors, which might be due to some uncharacterized compounding factors that were present when enlisting the participants. In addition, it is also plausible that in all regions, the diet, daily life, and training of athletes were properly arranged to be beneficial for the athletes' health, which possibly mitigated the impacts of other geo-environmental factors. Moreover, we were also faced with many restrictions in obtaining geological and environmental data, with the major difficulty being that the data were neither open to the public nor free. As a consequence, we could not fully utilize a large amount of important data and resources. Another challenge during the analyses was that our team lacked experienced researchers in spatial statistics, which caused us extraordinary difficulties in running the software. Nevertheless, we overcame the limitation and took full advantage of the newly developed technology of spatial statistical analysis to introduce crucial geo-environmental data into our investigation, which is beneficial for the future development of medical studies. As the era of big data approaches, we encourage scientists in the field to employ similar strategies to pursue real questions. Although it is difficult to obtain a clear cause-and-effect relationship in most cases, these approaches enable us to fully exploit the data and to elucidate any connection between a disease and environmental factors.

\section{Conclusion}

In summary, the abnormal bone density of athletes in this study is characterized by the spatial distribution characteristics of the eastern region higher than the western region, and the southern and northern regions higher than the central region. Appropriate temperature changes and a certain temperature difference can reduce the occurrence of abnormal bone density.

\section{Role of Funding Source}

This study was supported by the National Key R \& D Program of China (2018YFC1504404) and the Key projects of the Chinese people's liberation army logistics security applied basic research (BLB19J006).

\section{Conflicts of Interest}

The authors declare no conflicts of interest regarding the publication of this paper.

\section{References}

[1] Manolagas, S.C. (2000) Birth and Death of Bone Cells: Basic Regulatory Mechanisms and Implications for the Pathogenesis and Treatment of Osteoporosis. Endocrine Reviews, 21, 115-137. https://doi.org/10.1210/er.21.2.115 
[2] Raisz, L.G. (2005) Pathogenesis of Osteoporosis: Concepts, Conflicts, and Prospect. Journal of Clinical Investigation, 115, 3318-3325. https://doi.org/10.1172/JCI27071

[3] Chou, S.H. and LeBoff, M.S. (2017) Vertebral Imaging in the Diagnosis of Osteoporosis: A Clinician's Perspective. Current Osteoporosis Reports, 15, 509-520. https://doi.org/10.1007/s11914-017-0404-x

[4] Moynihan, R., Doust, J. and Henry, D. (2012) Preventing Overdiagnosis: How to Stop Harming the Healthy. BMJ, 28, 344. https://doi.org/10.1136/bmj.e3502

[5] McMillan, L.B., Zengin, A., Ebeling, P.R., et al. (2017) Prescribing Physical Activity for the Prevention and Treatment of Osteoporosis in Older Adults. Healthcare, 5, 85. https://doi.org/10.3390/healthcare5040085

[6] Willems, H.M.E., van den Heuvel, E.G.H.M., Schoemaker, R.J.W., et al. (2017) Diet and Exercise: A Match Made in Bone. Current Osteoporosis Reports, 15, 555-563. https://doi.org/10.1007/s11914-017-0406-8

[7] Wood, C.L. and Ahmed, S.F. (2017) Bone Protective Agents in Children. Archives of Disease in Childhood, 103, 503-508.

https://doi.org/10.1136/archdischild-2016-311820

[8] Coon, E.R., Quinonez, R.A., Moyer, V.A., et al. (2014) Overdiagnosis: How Our Compulsion for Diagnosis May Be Harming Children. Pediatrics, 134, 1013-1023. https://doi.org/10.1542/peds.2014-1778

[9] Iqbal, M.M. (2000) Osteoporosis: Epidemiology, Diagnosis, and Treatment. Southern Medical Journal, 93, 2-18. https://doi.org/10.1097/00007611-200001000-00001

[10] Cole, Z.A., Dennison, E.M. and Cooper, C. (2008) Osteoporosis Epidemiology Update. Current Rheumatology Reports, 10, 92-96.

https://doi.org/10.1007/s11926-008-0017-6

[11] Dhanwal, D.K., Cooper, C. and Dennison, E.M. (2010) Geographic Variation in Osteoporotic Hip Fracture Incidence: The Growing Importance of Asian Influences in Coming Decades. Journal Osteoporosis, 2010, Article ID: 757102. https://doi.org/10.4061/2010/757102

[12] Dhanwal, D.K., Dennison, E.M., Harvey, N.C., et al. (2011) Epidemiology of Hip Fracture: Worldwide Geographic Variation. Indian Journal of Orthopaedics, 45, 15-22. https://doi.org/10.4103/0019-5413.73656

[13] Fan, L.X., Wu, E.Q., Qu, X.C., et al. (2016) Distribution Characteristics of Spermophilus dauricus in Manchuria City in China in 2015 through "3S" Technology. Biomedical Environmental Sciences, 29, 603-608.

[14] Fan, L.X., Ning, B.A. and Liu, Y. (2017) Progress of Applied Research Using “3S" Technologies in Disease Surveillance. Journal of Preventive Medicine of Chinese People's Liberation Army, 35, 276-279.

[15] Zhou, X.N. (2009) Spatial Epidemiology. Science Press, Beijing.

[16] Sun, J., Huang, H., Xiao, G.X., et al. (2015) Spatial Distribution of Liver Cancer Incidence in Shenqiu County, Henan Province, China: A Spatial Analysis. Biomedical Environmental Sciences, 28, 214-218.

[17] Carey, D.E. and Golden, N.H. (2015) Bone Health in Adolescence. Adolescent Medicine: State of the Art Reviews, 26, 291-325.

[18] Tian, L. and Yu, X. (2017) Fat, Sugar, and Bone Health: A Complex Relationship. Nutrients, 9, 506. https://doi.org/10.3390/nu9050506

[19] Sanches, C.P., Vianna, A.G.D. and de Carvalho Barreto, F. (2017) The Impact of Type 2 Diabetes on Bone Metabolism. Diabetology \& Metabolic Syndrome, 9, 85. https://doi.org/10.1186/s13098-017-0278-1 
[20] Ito, M. and Tanaka, S. (2016) Bone Disorder and Nutrition. Clinical Calcium, 26, 375-383.

[21] Bi, X., Duan, X.Y., Chen, F., et al. (2016) Analysis on Human Bone Mineral Density in Armed Police Officers and Soldiers from Different Areas. Journal of Logistics University of PAP (Medical Sciences), 25, 795-798. 